\title{
Multiplicity of solutions for Neumann problems resonant at any eigenvalue
}

\author{
Leszek Gasiński and Nikolaos S. Papageorgiou
}

\begin{abstract}
We consider a semilinear Neumann problem with a reaction which is resonant both at $\pm \infty$ and at zero with respect to any eigenvalue, possibly the same one. Using the reduction method and Morse theory, we show that the problem has at least two nontrivial smooth solutions.
\end{abstract}

\section{Introduction}

Let $\Omega \subseteq \mathbb{R}^{N}$ be a bounded domain with a $C^{2}$-boundary $\partial \Omega$. In this paper, we study the following semilinear Neumann problem:

$$
\begin{cases}-\Delta u(z)=f(z, u(z)) & \text { in } \Omega, \\ \frac{\partial u}{\partial n}=0 & \text { on } \partial \Omega .\end{cases}
$$

Our goal is to prove a multiplicity theorem when resonance can occur with respect to any eigenvalue of the negative Neumann Laplacian (hereafter denoted by $-\Delta^{N}$ ) both at zero and at $\pm \infty$. Recently this situation was investigated by Gasiński-Papageorgiou [6], who assumed that

$$
\lim _{|\zeta| \rightarrow+\infty} \frac{f(z, \zeta)}{\zeta}
$$

exists and belongs to the spectrum of $-\Delta^{N}$ and that the eigenvalues with respect to which resonance occurs at infinity and at zero are different. No such restrictions are imposed here. Other multiplicity results for resonant Neumann problems can be found in work from Filippakis-Papageorgiou [5] (resonance at infinity with respect to the principal eigenvalue $\lambda_{0}=0$ ) and Tang [12] and Tang-Wu [13] (resonance occurring only at zero).

Our approach uses the reduction method due to Amann [2] and Morse theory.

Kyoto Journal of Mathematics, Vol. 54, No. 2 (2014), 259-269

DOI 10.1215/21562261-2642386, (C) 2014 by Kyoto University

Received September 7, 2012. Revised January 15, 2013. Accepted January 21, 2013.

2010 Mathematics Subject Classification: Primary 35J20; Secondary 35J60, 58E05.

Gasiński's and Papageorgiou's work partially supported by the Marie Curie International Research Staff Exchange Scheme Fellowship within the 7th European Community Framework Programme under grant no. 295118, the National Science Center of Poland under grant no. N N201 604640, the International Project cofinanced by the Ministry of Science and Higher Education of Republic of Poland under grant no. W111/7.PR/2012 and the National Science Center of Poland under Maestro Advanced Project no. DEC-2012/06/A/ST1/00262. 


\section{Mathematical background}

Let $X$ be a Banach space, and let $X^{*}$ be its topological dual. By $\langle\cdot, \cdot\rangle$ we denote the duality brackets for the pair $\left(X^{*}, X\right)$. For a given $\varphi \in C^{1}(X)$, we say that $\varphi$ satisfies the Palais-Smale condition if every sequence $\left\{x_{n}\right\}_{n \geq 1} \subseteq X$, such that $\left\{\varphi\left(x_{n}\right)\right\}_{n \geq 1} \subseteq \mathbb{R}$ is bounded and $\varphi^{\prime}\left(x_{n}\right) \longrightarrow 0$ in $X^{*}$, admits a strongly convergent subsequence. For $\varphi \in C^{1}(X)$ and $c \in \mathbb{R}$, we introduce the following sets:

$$
\begin{aligned}
\varphi^{c} & =\{x \in X: \varphi(x) \leq c\}, \\
K_{\varphi} & =\left\{x \in X: \varphi^{\prime}(x)=0\right\}, \\
K_{\varphi}^{c} & =\left\{x \in K_{\varphi}: \varphi(x)=c\right\} .
\end{aligned}
$$

If $\left(Y_{1}, Y_{2}\right)$ is a topological pair with $Y_{2} \subseteq Y_{1} \subseteq X$, then for every integer $k \geq 0$, by $H_{k}\left(Y_{1}, Y_{2}\right)$ we denote the $k$ th relative singular homology group with integer coefficients. The critical groups of $\varphi$ at an isolated $u \in K_{\varphi}^{c}$ are defined by

$$
C_{k}(\varphi, u)=H_{k}\left(\varphi^{c} \cap U, \varphi^{c} \cap U \backslash\{u\}\right) \quad \forall k \geq 0,
$$

where $U$ is a neighborhood of $u$ such that $K_{\varphi} \cap \varphi^{c} \cap U=\{u\}$. The excision property of singular homology implies that this definition is independent of the particular choice of the neighborhood $U$. Suppose that $\varphi \in C^{1}(X)$ satisfies the Palais-Smale condition, and assume that $\inf \varphi\left(K_{\varphi}\right)>-\infty$. Let $c<\inf \varphi\left(K_{\varphi}\right)$. The critical groups of $\varphi$ at infinity are defined by

$$
C_{k}(\varphi, \infty)=H_{k}\left(X, \varphi^{c}\right) \quad \forall k \geq 0 .
$$

The second deformation theorem (see, e.g., Papageorgiou-Kyritsi [11, p. 349]) implies that the above definition is independent of the particular level $c<$ $\inf \varphi\left(K_{\varphi}\right)$. Suppose that $K_{\varphi}$ is finite. We set

$$
\begin{aligned}
& M(t, x)=\sum_{k \geq 0} \operatorname{rank} C_{k}(\varphi, x) t^{k} \quad \forall t \in \mathbb{R}, x \in K_{\varphi}, \\
& P(t, \infty)=\sum_{k \geq 0} \operatorname{rank} C_{k}(\varphi, \infty) t^{k} \quad \forall t \in \mathbb{R} .
\end{aligned}
$$

We have the Morse relation, which says that

$$
\sum_{x \in K_{\varphi}} M(t, x)=P(t, \infty)+(1+t) Q(t)
$$

where

$$
Q(t)=\sum_{k \geq 1} \beta_{k} t^{k}
$$

is a formal series in $t \in \mathbb{R}$ with nonnegative integer coefficients $\beta_{k}$ (see Chang [3]). 


\section{Multiplicity theorem}

In what follows, by $\left\{\widehat{\lambda}_{k}\right\}_{k \geq 0}$ we denote the distinct eigenvalues of $-\Delta^{N}$. Recall that $\widehat{\lambda}_{0}=0$ is the principal eigenvalue. By $E\left(\widehat{\lambda}_{k}\right)$ we denote the corresponding eigenspace.

The hypotheses on the reaction $f(z, \zeta)$ are the following:

$\underline{H}: f: \Omega \times \mathbb{R} \longrightarrow \mathbb{R}$ is a measurable function such that $f(z, 0)=0$ and $f(z, \cdot) \in$ $C^{1}(\mathbb{R})$ for almost all $z \in \Omega$ and

(i) there exists $\eta \in L^{\infty}(\Omega)_{+}, \eta(z) \leq \widehat{\lambda}_{m+1}$ for almost all $z \in \Omega, \eta \neq \widehat{\lambda}_{m+1}$ with $m \geq 0$, such that

$$
\left|f_{\zeta}^{\prime}(z, \zeta)\right| \leq \eta(z) \quad \text { for almost all } z \in \Omega, \text { all } \zeta \in \mathbb{R}
$$

(ii) we have

$$
\widehat{\lambda}_{m} \leq \liminf _{\zeta \rightarrow \pm \infty} \frac{f(z, \zeta)}{\zeta} \quad \text { uniformly for almost all } z \in \Omega
$$

and if

$$
F(z, \zeta)=\int_{0}^{\zeta} f(z, s) d s
$$

then

$$
f(z, \zeta) \zeta-2 F(z, \zeta) \longrightarrow-\infty \quad \text { as } \zeta \rightarrow \pm \infty
$$

uniformly for almost all $z \in \Omega$;

(iii) there exist integer $l \geq 0$ and $\delta>0$ such that

$$
\widehat{\lambda}_{l} \leq \frac{f(z, \zeta)}{\zeta} \leq \widehat{\lambda}_{l+1} \text { for almost all } z \in \Omega, \text { all } 0<|\zeta| \leq \delta
$$

\section{REMARK 3.1}

From hypotheses $H(\mathrm{i})$ and $H(\mathrm{ii})$ and the mean value theorem, we have

$$
\widehat{\lambda}_{m} \leq \liminf _{\zeta \rightarrow \pm \infty} \frac{f(z, \zeta)}{\zeta} \leq \limsup _{\zeta \rightarrow \pm \infty} \frac{f(z, \zeta)}{\zeta} \leq \eta(z)
$$

uniformly for almost all $z \in \Omega$. So resonance can occur at $\pm \infty$ with respect to any eigenvalue $\widehat{\lambda}_{m}, m \geq 0$. However, note that we do not require that the quotient $f(z, \zeta) / \zeta$ necessarily has a limit as $\zeta \longrightarrow \pm \infty$.

Similarly, hypothesis $H$ (iii) permits resonance at zero to any eigenvalue $\widehat{\lambda}_{l}$, $l \geq 0$, which need not be distinct from $\widehat{\lambda}_{m}$.

Let $\varphi: H^{1}(\Omega) \longrightarrow \mathbb{R}$ be the energy functional for problem (1.1), defined by

$$
\varphi(u)=\frac{1}{2}\|\nabla u\|_{2}^{2}-\int_{\Omega} F(z, u(z)) d z \quad \forall u \in H^{1}(\Omega) .
$$


Evidently $\varphi \in C^{2}\left(H^{1}(\Omega)\right)$. Also, we consider the following orthogonal direct sum decomposition of $H^{1}(\Omega)$ :

$$
H^{1}(\Omega)=Y \oplus \widehat{H} \quad \text { with } Y=\bigoplus_{i=0}^{m} E\left(\widehat{\lambda}_{i}\right), \widehat{H}=Y^{\perp}
$$

PROPOSITION 3.2

If hypotheses $H$ hold, then there exists a $C^{1}$-map $\vartheta: Y \longrightarrow \widehat{H}$ such that

$$
\inf _{\widehat{u} \in \widehat{H}} \varphi(y+\widehat{u})=\varphi(y+\vartheta(y)) \quad \forall y \in Y .
$$

Proof

We fix $y \in Y$, and let $\varphi_{y}: H^{1}(\Omega) \longrightarrow \mathbb{R}$ be the $C^{2}$-functional defined by

$$
\varphi_{y}(u)=\varphi(y+u) \quad \forall u \in H^{1}(\Omega) .
$$

Let $i: \widehat{H} \longrightarrow H^{1}(\Omega)$ be the inclusion map, and let $\widehat{\varphi}_{y}: \widehat{H} \longrightarrow \mathbb{R}$ be defined by

$$
\widehat{\varphi}_{y}=\varphi_{y} \circ i \text {. }
$$

Recall that $i^{*}=p_{\widehat{H}^{*}}$ (the orthogonal projection on $\left.\widehat{H}^{*}\right)$. From the chain rule, we have

$$
\widehat{\varphi}_{y}^{\prime}(\widehat{u})=p_{\widehat{H}^{*}} \varphi_{y}^{\prime}(\widehat{u})=p_{\widehat{H}^{*}}\left(A(y+\widehat{u})-N_{f}(y+\widehat{u})\right),
$$

where $A \in \mathcal{L}\left(H^{1}(\Omega) ; H^{1}(\Omega)^{*}\right)$ is defined by

$$
\langle A(u), v\rangle=\int_{\Omega}(\nabla u, \nabla v)_{\mathbb{R}^{N}} d z \quad \forall u, v \in H^{1}(\Omega)
$$

and

$$
N_{f}(u)(\cdot)=f(\cdot, u(\cdot)) \quad \forall u \in H^{1}(\Omega) .
$$

Let $\langle\cdot, \cdot\rangle_{\widehat{H}}$ denote the duality brackets for the pair $\left(\widehat{H}^{*}, \widehat{H}\right)$, and let $\langle\cdot, \cdot\rangle$ denote the duality brackets for the pair $\left(H^{1}(\Omega)^{*}, H^{1}(\Omega)\right)$. For $\widehat{u}_{1}, \widehat{u}_{2} \in \widehat{H}$, we have

$$
\begin{aligned}
\left\langle\widehat{\varphi}_{y}^{\prime}\left(\widehat{u}_{1}\right)-\widehat{\varphi}_{y}^{\prime}\left(\widehat{u}_{2}\right), \widehat{u}_{1}-\widehat{u}_{2}\right\rangle_{\widehat{H}} & =\left\langle A\left(\widehat{u}_{1}\right)-A\left(\widehat{u}_{2}\right), \widehat{u}_{1}-\widehat{u}_{2}\right\rangle \\
& \quad-\int_{\Omega}\left(f\left(z, y+\widehat{u}_{1}\right)-f\left(z, y+\widehat{u}_{2}\right)\right)\left(\widehat{u}_{1}-\widehat{u}_{2}\right) d z .
\end{aligned}
$$

Hypothesis $H_{1}(\mathrm{i})$ and the mean value theorem imply that

$$
\left|f\left(z,\left(y+\widehat{u}_{1}\right)(z)\right)-f\left(z,\left(y+\widehat{u}_{2}\right)(z)\right)\right| \leq \eta(z)\left|\widehat{u}_{1}(z)-\widehat{u}_{2}(z)\right|
$$

for almost all $z \in \Omega$. Using this in (3.1), we obtain

$$
\begin{aligned}
\left\langle\widehat{\varphi}_{y}^{\prime}\left(\widehat{u}_{1}\right)-\widehat{\varphi}_{y}^{\prime}\left(\widehat{u}_{2}\right), \widehat{u}_{1}-\widehat{u}_{2}\right\rangle_{\widehat{H}} & \geq\left\|\nabla\left(\widehat{u}_{1}-\widehat{u}_{2}\right)\right\|_{2}^{2}-\int_{\Omega} \eta(z)\left|\widehat{u}_{1}-\widehat{u}_{2}\right|^{2} d z \\
& \geq \xi_{0}\left\|\widehat{u}_{1}-\widehat{u}_{2}\right\|^{2}
\end{aligned}
$$

for some $\xi_{0}>0$ (see Gasiński-Papageorgiou [6, Proposition 2.5]), so $\widehat{\varphi}_{y}$ is strictly convex. 
Also, we have

$$
\left\langle\widehat{\varphi}_{y}^{\prime}(\widehat{u}), \widehat{u}\right\rangle=\left\langle\widehat{\varphi}_{y}^{\prime}(\widehat{u})-\widehat{\varphi}_{y}^{\prime}(0), \widehat{u}\right\rangle+\left\langle\widehat{\varphi}_{y}^{\prime}(0), \widehat{u}\right\rangle \geq \xi_{0}\|\widehat{u}\|^{2}-c_{1}\|\widehat{u}\|,
$$

for some $c_{1}>0$ (see (3.1)), so

$$
\text { the map } \widehat{u} \longmapsto \widehat{\varphi}_{y}^{\prime}(\widehat{u}) \text { is coercive on } \widehat{H} \text {. }
$$

But $\widehat{\varphi}_{y}^{\prime}$ is maximal monotone (see Filippakis-Papageorgiou [5]). Hence, it is surjective (see Papageorgiou-Kyritsi [11, Corollary 3.2.28, p. 172]). So, we can find $\widehat{u}_{0} \in \widehat{H}$ such that

$$
\widehat{\varphi}_{y}^{\prime}\left(\widehat{u}_{0}\right)=0
$$

From the strict convexity of $\widehat{\varphi}_{y}$, it follows that $\widehat{u}_{0}$ is the unique global minimizer of $\widehat{\varphi}_{y}$. Let $\vartheta: Y \longrightarrow \widehat{H}$ be the map that assigns to each $y \in Y$ the unique minimizer $\widehat{u}_{0}=\widehat{u}_{0}(y)$ of $\widehat{\varphi}_{y}$. We have

$$
\widehat{\varphi}_{y}^{\prime}(\vartheta(y))=p_{\widehat{H}^{*}} \varphi^{\prime}(y+\vartheta(y)) \quad \text { and } \quad \varphi(y+\vartheta(y))=\inf _{\widehat{u} \in \widehat{H}} \varphi(y+\widehat{u}) .
$$

Let $\sigma: Y \times \widehat{H} \longrightarrow \widehat{H}^{*}$ be defined by

$$
\sigma(y, \widehat{u})=p_{\widehat{H}^{*}} \varphi^{\prime}(y+\widehat{u}) \quad \forall(y, \widehat{u}) \in Y \times \widehat{H} .
$$

If $\sigma\left(y_{0}, \widehat{u}_{0}\right)=0$, then from (3.5), we have that $\widehat{u}_{0}=\vartheta\left(y_{0}\right)$. Recall that $\varphi \in$ $C^{2}\left(H^{1}(\Omega)\right)$. So, from (3.6), it is clear that $\sigma \in C^{1}\left(Y \times \widehat{H}, \widehat{H}^{*}\right)$, and for all $\widehat{u} \in \widehat{H}$, we have

$$
\begin{aligned}
\left\langle\sigma_{\widehat{u}}^{\prime}\left(y_{0}, \widehat{u}_{0}\right) \widehat{u}, \widehat{u}\right\rangle_{\widehat{H}} & =\left\langle p_{\widehat{H}^{*}} \varphi^{\prime \prime}\left(y_{0}+\widehat{u}_{0}\right) \widehat{u}, \widehat{u}\right\rangle_{\widehat{H}} \\
& =\left\langle\varphi^{\prime \prime}\left(y_{0}+\widehat{u}_{0}\right) \widehat{u}, \widehat{u}\right\rangle \\
& =\|\nabla \widehat{u}\|_{2}^{2}-\int_{\Omega} f_{\zeta}^{\prime}\left(z, y_{0}+\widehat{u}_{0}\right) \widehat{u}^{2} d z \\
& \geq\|\nabla \widehat{u}\|_{2}^{2}-\int_{\Omega} \eta \widehat{u}^{2} d z \\
& \geq \xi_{0}\|\widehat{u}\|^{2},
\end{aligned}
$$

where we used the orthogonality of $Y$ and $\widehat{H}$, hypothesis $H(\mathrm{i})$, and [6, Proposition 2.5]. So $\sigma_{\widehat{u}}^{\prime}\left(y_{0}, \widehat{u}_{0}\right) \in \mathcal{L}\left(\widehat{H}, \widehat{H}^{*}\right)$ is an isomorphism.

Invoking the implicit function theorem, we conclude that $\vartheta \in C^{1}(Y ; \widehat{H})$.

Let $\bar{\varphi}: Y \longrightarrow \mathbb{R}$ be defined by

$$
\bar{\varphi}(y)=\varphi(y+\vartheta(y)) \quad \forall y \in Y .
$$

\section{PROPOSITION 3.3}

If hypotheses $H$ hold, then $\bar{\varphi}$ is anticoercive; that is, if $\|y\| \longrightarrow \infty$, then $\bar{\varphi}(y) \longrightarrow$ $-\infty$. 
Proof

We argue by contradiction. So, suppose that the result is not true. Then we can find $M>0$ and a sequence $\left\{y_{n}\right\}_{n \geq 1} \subseteq Y$ such that

$$
\left\|y_{n}\right\| \longrightarrow+\infty \quad \text { and } \quad-M \leq \bar{\varphi}\left(y_{n}\right) \quad \forall n \geq 1 .
$$

From the definition of $\bar{\varphi}$ (see $(3.7)$ ), we have

$$
\begin{aligned}
-M & \leq \bar{\varphi}\left(y_{n}\right) \leq \varphi\left(y_{n}\right)=\frac{1}{2}\left\|\nabla y_{n}\right\|_{2}^{2}-\int_{\Omega} F\left(z, y_{n}\right) d z \\
& \leq \int_{\Omega}\left(\frac{1}{2} \lambda_{m} y_{n}^{2}-F\left(z, y_{n}\right)\right) d z
\end{aligned}
$$

since $y_{n} \in Y$. Let

$$
\widehat{f}(z, \zeta)=f(z, \zeta)-\widehat{\lambda}_{m} \zeta
$$

and let

$$
\widehat{F}(z, \zeta)=\int_{0}^{\zeta} \widehat{f}(z, s) d s
$$

Then

$$
0 \leq \liminf _{\zeta \rightarrow \pm \infty} \frac{\widehat{f}(z, \zeta)}{\zeta} \leq \limsup _{\zeta \rightarrow \pm \infty} \frac{\widehat{f}(z, \zeta)}{\zeta} \leq \eta(z)-\widehat{\lambda}_{m}
$$

uniformly for almost all $z \in \Omega$, so

$$
0 \leq \liminf _{\zeta \rightarrow \pm \infty} \frac{2 \widehat{F}(z, \zeta)}{\zeta^{2}} \leq \limsup _{\zeta \rightarrow \pm \infty} \frac{2 \widehat{F}(z, \zeta)}{\zeta^{2}} \leq \eta(z)-\widehat{\lambda}_{m}
$$

uniformly for almost all $z \in \Omega$ (see [1, Remark 26]).

Also, we have (see hypothesis $H(\mathrm{ii})$ )

$$
\lim _{\zeta \rightarrow \pm \infty}(\widehat{f}(z, \zeta) \zeta-2 \widehat{F}(z, \zeta))=-\infty \quad \text { uniformly for almost all } z \in \Omega .
$$

Let

$$
v_{n}=\frac{y_{n}}{\left\|y_{n}\right\|} \quad \forall n \geq 1
$$

Then

$$
\left\|v_{n}\right\|=1 \quad \forall n \geq 1
$$

and by virtue of the finite dimensionality of $Y$, passing to a subsequence if necessary, we may assume that

$$
v_{n} \longrightarrow v \text { in } H^{1}(\Omega),
$$

with $\|v\|=1$. Hence,

$$
|\{v \neq 0\}|_{N}>0
$$

where $|\cdot|_{N}$ denotes the Lebesgue measure on $\mathbb{R}^{N}$, and

$$
\left|y_{n}(z)\right| \longrightarrow+\infty \text { for almost all } z \in\{v \neq 0\} \text {. }
$$


By virtue of (3.10), for a given $\beta>0$, we can find $\widehat{M}=\widehat{M}(\beta)>0$ such that

$$
\widehat{f}(z, \zeta) \zeta-2 \widehat{F}(z, \zeta) \leq-\beta \text { for almost all } z \in \Omega \text {, all }|\zeta| \geq \widehat{M} .
$$

For almost all $z \in \Omega$ and all $\zeta \geq \widehat{M}$, we have (see (3.20))

$$
\frac{d}{d \zeta} \frac{\widehat{F}(z, \zeta)}{\zeta^{2}}=\frac{\widehat{f}(z, \zeta) \zeta-2 \widehat{F}(z, \zeta)}{\zeta^{3}} \leq-\frac{\beta}{\zeta^{3}} .
$$

Integrating (3.12) on $[\zeta, y]$ (with $\widehat{M} \leq \zeta \leq y$ ), we have

$$
\frac{\widehat{F}(z, y)}{y^{2}}-\frac{\widehat{F}(z, \zeta)}{\zeta^{2}} \leq \frac{\beta}{2}\left(\frac{1}{y^{2}}-\frac{1}{\zeta^{2}}\right) \text { for almost all } z \in \Omega .
$$

We let $y \longrightarrow+\infty$ and obtain (see (3.9))

so

$$
-\frac{\widehat{F}(z, \zeta)}{\zeta^{2}} \leq-\frac{\beta}{2 \zeta^{2}} \quad \text { for almost all } z \in \Omega, \text { all } \zeta \geq \widehat{M},
$$

$$
F(z, \zeta) \geq \frac{\beta}{2} \quad \text { for almost all } z \in \Omega, \text { all } \zeta \geq \widehat{M}
$$

Since $\beta>0$ is arbitrary, we conclude that $F(z, \zeta) \longrightarrow+\infty$ as $\zeta \rightarrow+\infty$ uniformly for almost all $z \in \Omega$.

Similarly, we show that $\widehat{F}(z, \zeta) \longrightarrow+\infty$ as $\zeta \rightarrow-\infty$. Then, from (3.8), we have

$$
-M \leq \bar{\varphi}\left(y_{n}\right) \leq-\int_{\Omega} \widehat{F}\left(z, y_{n}\right) d z \longrightarrow-\infty
$$

(recall that $\left|y_{n}(z)\right| \rightarrow+\infty$ for almost all $z \in\{v \neq 0\}$ ), a contradiction.

We assume that $K_{\bar{\varphi}}$ is finite. (Otherwise we already have a whole sequence of nontrivial solutions of (1.1) and so we are done.) From Proposition 3.3, we know that $-\bar{\varphi}$ is coercive; hence, it satisfies the Palais-Smale condition. Therefore, $\bar{\varphi}$ satisfies the Palais-Smale condition, and we can consider the critical groups of $\bar{\varphi}$ at infinity. In fact, using ideas from Liu [9], we can compute exactly these critical groups of $\bar{\varphi}$.

\section{PROPOSITION 3.4}

If hypotheses $H$ hold, then

$$
C_{k}(\bar{\varphi}, \infty)=\delta_{k, d_{m}} \mathbb{Z} \quad \forall k \geq 0,
$$

with $d_{m}=\operatorname{dim} Y \geq 1$.

Proof

Let $m_{0}<\inf \bar{\varphi}\left(K_{\bar{\varphi}}\right)$. Since $\bar{\varphi}$ is anticoercive (see Proposition 3.3), we can find $\gamma<\beta<m_{0}$ and $0<\varrho<R$ such that

$$
C_{r} \subseteq \bar{\varphi}^{\gamma} \subseteq C_{\varrho} \subseteq \bar{\varphi}^{\beta},
$$

where, for every $r>0, C_{r}=\{y \in Y:\|y\| \geq r\}$. 
For the triples $\left(C_{R}, C_{\varrho}, Y\right)$ and $\left(\bar{\varphi}^{\gamma}, \bar{\varphi}^{\beta}, Y\right)$ we consider the corresponding long exact sequences of homology groups. So, we have

$$
\begin{array}{ccc}
H_{k}\left(C_{\varrho}, C_{R}\right) & \stackrel{\left.h_{*}\right|_{C_{\varrho}}}{\longrightarrow} & H_{k}\left(\bar{\varphi}^{\beta}, \bar{\varphi}^{\gamma}\right) \\
\downarrow i^{*} & \downarrow \widehat{i}_{*} \\
H_{k}\left(Y, C_{R}\right) & \stackrel{h_{*}}{\longrightarrow} & H_{k}\left(Y, \bar{\varphi}^{\gamma}\right) \\
\downarrow j_{*} & \downarrow \widehat{j}_{*} \\
H_{k}\left(Y, C_{\varrho}\right) & \stackrel{h_{*}}{\longrightarrow} & H_{k}\left(Y, \bar{\varphi}^{\beta}\right) \\
\downarrow \partial_{*} & & \downarrow \widehat{\partial}_{*} \\
H_{k-1}\left(C_{\varrho}, C_{R}\right) \stackrel{\left.h_{*}\right|_{C}}{\longrightarrow} & H_{k-1}\left(\bar{\varphi}^{\beta}, \bar{\varphi}^{\gamma}\right)
\end{array}
$$

In (3.13) all squares are commutative (see Granas-Dugundji [7, p. 377]) and the maps $i_{*}, j_{*}, \widehat{i}_{*}, \widehat{j}_{*}, h_{*}$ are the group homomorphisms induced by the corresponding inclusion maps. Finally, $\partial_{*}$ and $\widehat{\partial}_{*}$ are the corresponding boundary homomorphisms.

Since $\gamma<\beta<m_{0}<\inf \bar{\varphi}\left(K_{\bar{\varphi}}\right)$, we have

$$
H_{k}\left(\bar{\varphi}^{\beta}, \bar{\varphi}^{\gamma}\right)=0 \quad \forall k \geq 0
$$

by the second deformation theorem (see Papageorgiou-Kyritsi [11, Theorem 4.6.1, p. 349]). We consider the map $\xi: C_{\varrho} \longrightarrow C_{R}$, defined by

$$
\xi(u)= \begin{cases}R \frac{u}{\|u\|} & \text { if } \varrho \leq\|u\| \leq R, \\ u & \text { if } R<\|u\| .\end{cases}
$$

Evidently $\xi$ is continuous and $\left.\xi\right|_{C_{R}}=\left.i d\right|_{C_{R}}$. Therefore, $C_{R}$ is a retract of $C_{\varrho}$. Moreover, if $h:[0,1] \times C_{\varrho} \longrightarrow Y$ is defined by

$$
h(t, u)=(1-t) u+t R \frac{u}{\|u\|} \quad \forall(t, u) \in[0,1] \times C_{\varrho},
$$

then we see that $C_{\varrho}$ is deformable into $C_{R}$ over $Y$. So, invoking [4, Theorem 6.5, p. 325], we have that $C_{R}$ is a deformation retract of $C_{\varrho}$. Hence (see GranasDugundji [7, p. 387]),

$$
H_{k}\left(C_{\varrho}, C_{R}\right)=0 \quad \forall k \geq 0
$$


From the exactness of the long homology sequence in (3.13), we have (see (3.15))

$$
0=\operatorname{im} i_{*}=\operatorname{ker} j_{*} \quad \text { and } \quad \operatorname{im} j_{*}=\operatorname{ker} \partial_{*}=H_{k}\left(Y, C_{\varrho}\right)
$$

and (see (3.14))

$$
0=\operatorname{im} \widehat{i}_{*}=\operatorname{ker} \widehat{j}_{*} \quad \text { and } \quad \operatorname{im} \widehat{j}_{*}=\operatorname{ker} \widehat{\partial}_{*}=H_{k}\left(Y, \bar{\varphi}^{\beta}\right) .
$$

From these equalities, it follows that both $j_{*}$ and $\widehat{j}_{*}$ are group homomorphisms. Then invoking $\left[7\right.$, Lemma D.1, p. 610], we infer that $h_{*}$ is an isomorphism. So, we have

$$
H_{k}\left(Y, C_{\varrho}\right)=H_{k}\left(Y, \bar{\varphi}^{\beta}\right)
$$

hence,

$$
H_{k}\left(Y, C_{\varrho}\right)=C_{k}(\bar{\varphi}, \infty) \quad \forall k \geq 0 .
$$

Recall that $\beta<\inf \bar{\varphi}\left(K_{\bar{\varphi}}\right)$.

As before, using the radial retraction and [4, Theorem 6.5, p. 325], we show that $\partial B_{\varrho}=\{y \in Y:\|y\|=\varrho\}$ is a deformation retract of $C_{\varrho}$. Therefore,

$$
H_{k}\left(Y, C_{\varrho}\right)=H_{k}\left(Y, \partial B_{\varrho}\right) \quad \forall k \geq 0,
$$

so (see Maunder [10, Example 4.3.12, pp. 120-121])

$$
H_{k}\left(Y, C_{\varrho}\right)=\delta_{k, d_{m}} \mathbb{Z} \quad \forall k \geq 0,
$$

and thus (see (3.16))

$$
C_{k}(\bar{\varphi}, \infty)=\delta_{k, d_{m}} \mathbb{Z} \quad \forall k \geq 0
$$

Now, we are ready for the multiplicity theorem.

\section{THEOREM 3.5}

If hypotheses $H$ hold, then problem (1.1) has at least two nontrivial smooth solutions $u_{0}, \widehat{u} \in C^{1}(\bar{\Omega})$.

\section{Proof}

Since $\varphi \in C^{2}\left(H^{1}(\Omega)\right)$ and $\vartheta \in C^{1}(Y ; \widehat{H})$ (see Proposition 3.2), it follows that $\bar{\varphi} \in C^{1}(Y)$ (see $(3.7)$ ). Also, from Proposition 3.3, we know that $\bar{\varphi}$ is anticoercive. Since $Y$ is finite-dimensional, we can find $y_{0} \in Y$ such that

$$
\bar{\varphi}\left(y_{0}\right)=\max \{\bar{\varphi}(y): y \in Y\} \text {. }
$$

Then from Chang [3, Example 1, p. 33], we have

$$
C_{k}\left(\bar{\varphi}, y_{0}\right)=\delta_{k, d_{m}} \mathbb{Z} \quad \forall k \geq 0 .
$$

Hypothesis $H$ (iii) implies that

$$
C_{k}(\varphi, 0)=\delta_{k, d_{l}} \mathbb{Z} \quad \forall k \geq 0,
$$


where

$$
d_{l}=\operatorname{dim} \bigoplus_{i=0}^{l} E\left(\widehat{\lambda}_{i}\right) .
$$

This can be established as in the work of Li-Perera-Su [8, Proposition 1.1], who deal with Dirichlet spaces. Since their proof uses only the homotopy invariance of critical groups and the orthogonal direct sum decomposition of the ambient space, it carries also to the Neumann case. From (3.18) and since $\vartheta(0)=0$ (see (3.3) and (3.5)), we have (see Liu [9, Lemma 2.3, p. 501])

$$
C_{k}(\bar{\varphi}, 0)=\delta_{k, d_{\varrho}} \mathbb{Z} \quad \forall k \geq 0 .
$$

Finally, from Proposition 3.4, we have

$$
C_{k}(\bar{\varphi}, \infty)=\delta_{k, d_{m}} \mathbb{Z} \quad \forall k \geq 0 .
$$

If $K_{\bar{\varphi}}=\left\{0, y_{0}\right\}$, then from (3.17), (3.19), and (3.20) and the Morse relation with $t=-1$ (see $(2.1)$ ), we have

$$
(-1)^{d_{l}}=0
$$

a contradiction. So, $\bar{\varphi}$ has at least one more critical point $\widehat{y} \notin\left\{0, y_{0}\right\}$. Let $u_{0}=$ $y_{0}+\vartheta\left(y_{0}\right)$, and let $\widehat{u}=\widehat{y}+\vartheta(\widehat{y})$. Then $u_{0}, \widehat{u}$ are solutions of $(1.1)$, and by standard regularity theory, we have $u_{0}, \widehat{u} \in C^{1}(\bar{\Omega})$.

\section{References}

[1] S. Aizicovici, N. S. Papageorgiou, and V. Staicu, Degree Theory for Operators of Monotone Type and Nonlinear Elliptic Equations with Inequality Constraints, Mem. Amer. Math. Soc. 196, Amer. Math. Soc., Providence, 2008. MR 2459421. DOI 10.1090/memo/0915.

[2] H. Amann, Saddle points and multiple solutions of differential equations, Math. Z. 169 (1979), 127-166. MR 0550724. DOI 10.1007/BF01215273.

[3] K.-C. Chang, Infinite-Dimensional Morse Theory and Multiple Solution Problems, Progr. Nonlinear Differential Equations Appl. 6, Birkhäuser, Boston, 1993. MR 1196690.

[4] J. Dugundji, Topology, Allyn and Bacon, Boston, 1966. MR 0193606.

[5] M. Filippakis and N. S. Papageorgiou, Multiple nontrivial solutions for resonant Neumann problems, Math. Nachr. 283 (2010), 1000-1014.

MR 2677284. DOI 10.1002/mana.200710045.

[6] L. Gasiński and N. S. Papageorgiou, Neumann problems resonant at zero and infinity, Ann. Mat. Pura Appl. (4) 191 (2012), 395-430. MR 2958341. DOI 10.1007/s10231-011-0188-z.

[7] A. Granas and J. Dugundji, Fixed Point Theory, Springer Monogr. Math., Springer, New York, 2003. MR 1987179.

[8] S. Li, K. Perera, and J. Su, Computation of critical groups in elliptic boundary value problems where the asymptotic limits may not exist, Proc. Roy. Soc. 
Edinburgh Sect. A 131 (2001), 721-732. MR 1838509.

DOI $10.1017 / \mathrm{S} 0308210500001074$.

[9] S. Liu, Remarks on multiple solutions for elliptic resonant problems, J. Math. Anal. Appl. 336 (2007), 498-505. MR 2348521.

DOI 10.1016/j.jmaa.2007.01.051.

[10] C. R. F. Maunder, Algebraic Topology, Cambridge Univ. Press, Cambridge, 1980. MR 0694843.

[11] N. S. Papageorgiou and S. T. Kyritsi-Yiallourou, Handbook of Applied Analysis, Adv. Mech. Math. 19, Springer, New York, 2009. MR 2527754.

DOI $10.1007 / \mathrm{b} 120946$.

[12] C.-L. Tang, Multiple solutions of Neumann problems for elliptic equations, Nonlinear Anal. 54 (2003), 637-650. MR 1983440.

DOI 10.1016/S0362-546X(03)00091-9.

[13] C.-L. Tang and X.-P. Wu, Existence and multiplicity for solutions of Neumann problem for elliptic equations, J. Math. Anal. Appl. 288 (2003), 660-670.

MR 2020187. DOI 10.1016/j.jmaa.2003.09.034.

Gasiński: Faculty of Mathematics and Computer Science, Jagiellonian University, ulica Łojasiewicza 6, 30-348 Kraków, Poland; Leszek.Gasinski@ii.uj.edu.pl

Papageorgiou: Department of Mathematics, National Technical University, Zografou Campus, Athens 15780, Greece; npapg@math.ntua.gr 